\title{
Smartphone apps for managing alcohol consumption: a literature review
}

\author{
Stephanie Colbert ${ }^{1}$, Louise Thornton ${ }^{2}$ and Robyn Richmond ${ }^{1 *}$ (B)
}

\begin{abstract}
Background: Smartphone applications (apps) designed to assist users to reduce hazardous and harmful alcohol consumption show potential as an inexpensive alternative to traditional brief intervention in primary care. The aim of this paper is to provide an overview of the literature on alcohol reduction apps and the availability of evidencedbased apps on top commercial app stores.

Methods: We reviewed literature through to December 2019 using the databases PubMed, MEDLINE, PsycINFO and Google Scholar and keyword search terms smartphone/mobile/phone AND application/app AND alcohol. Articles were included if the primary intervention was a smartphone app and the study measured participant changes in frequency or volume of alcohol consumption.

Results: 21 relevant articles were identified that evaluated 19 unique smartphone apps. Of the 19 unique apps, seven were designed for use among youth and 12 in adult populations. The available evidence for the efficacy of alcohol reduction apps among youth is inconclusive, with results from these evaluations not showing a clear benefit in reducing alcohol consumption compared to control groups. The results of apps designed for adult populations appears more promising, but results are still mixed. Of the 19 alcohol reduction apps that have been evaluated only eight of these are currently publicly available in commercial app stores. Of these eight apps, only four were demonstrated in the literature to assist with reducing alcohol consumption.
\end{abstract}

Conclusion: The evidence for alcohol reduction apps is promising but inconclusive. Few apps that have been evaluated in the scientific literature are currently available for download in commercial app stores.

Keywords: Smartphone, Mobile phone, Apps, Application, Alcohol, Drinking

\section{Introduction}

Alcohol use is a leading risk factor for death and disability, accounting for $5.1 \%$ of the global burden of disease [1]. Alcohol use has been linked to over 200 health conditions, including cardiovascular disease, cancers, dementia, road injuries, violence and suicides [1]. Despite the risk of adverse consequences, many people still consume alcohol at levels that increase their risk of harm [1-3].

*Correspondence: r.richmond@unsw.edu.au

1 School Public Health and Community Medicine, University of New South Wales, Kensington, Australia

Full list of author information is available at the end of the article
Face-to-face brief interventions delivered in primary care appear to be effective in reducing alcohol consumption in patients drinking at hazardous and harmful levels [4]. A 2018 Cochrane review found that hazardous or harmful drinkers who received brief intervention consumed a mean of 20 grams per week less alcohol (95\% $\mathrm{CI}=28$ to 12 ) after 1 year than participants who received minimal or no intervention [4]. However, several barriers exist which limit the reach of brief interventions in primary care, including heavy clinician workload, a lack of appropriate training and limited time in consultations to manage competing health priorities [5]. For patients, barriers for not seeking treatment include the stigma 
associated with substance use disorders, and logistical and financial barriers [6].

The use of digital interventions (e.g. websites and smartphone applications), has the potential to overcome some of the barriers by offering an inexpensive, accessible method to deliver education, support and monitoring through personal devices. There have been several metaanalyses conducted evaluating the effectiveness of digital interventions for reducing alcohol consumption that have consistently found small reductions in the intervention groups compared to controls [7-9]. A 2017 Cochrane review reported that participants using a digital intervention drank approximately 23 grams of alcohol per week less than controls who received information only or usual care [10].

In more recent years the focus in digital health has shifted to smartphone apps, with more than 318,000 health apps currently available on top app stores and an estimated 200 new apps being added daily [11]. Smartphone apps have several advantages over standard websites, including the ability to be used without internet access and the ability to provide a more personalised experience for users with options for customization. With near ubiquitous smartphone ownership in countries such as the United States [12] and Australia [13], if effective, these apps have great potential to improve health outcomes.

There are a large number of apps currently available in commercial app stores that are aimed at assisting users to reduce their alcohol intake, however few have been evaluated. The aim of this paper is to provide an overview of the literature on alcohol reduction apps and the availability of evidenced-based apps on top commercial app stores.

\section{Method}

We conducted a literature review of English language articles published until 4 December 2019 which evaluated the efficacy or effectiveness of a smartphone app in reducing alcohol consumption. The databases searched were PubMed, MEDLINE, PsycINFO and Google Scholar. Keyword search terms were smartphone/mobile/phone AND application/app AND alcohol. Titles and abstracts of all potentially relevant articles were reviewed for possible inclusion by one author (SC). Articles were included if the primary intervention was a smartphone app (native app not a web-based app) and the study measured participant changes in frequency or volume of alcohol consumption. Reference lists from each article were also examined to identify articles that may have been missed in the initial search.

For each unique app identified in the papers we searched for its availability in the top two app stores,
Google Play and iTunes in January-February 2020. We searched the United States (US), Canadian, United Kingdom (UK), Irish, Australian and New Zealand (NZ) iTunes and Google Play stores for all apps, plus the iTunes and Google Play stores of the country where the app was developed, e.g. if the app was developed in Sweden we also searched the Swedish iTunes and Google Play stores. If the app could not be found in any Google Play or iTunes stores the corresponding author of the study was emailed to enquire about the app's availability.

\section{Results}

The database searches returned 518 results of which 21 were studies evaluating whether use of a smartphone app reduced participants' frequency or volume of alcohol consumption [14-34]. These 21 articles evaluated 19 unique smartphone apps. Eight of these apps could be found in the iTunes and/or Google Play stores of at least one of the following countries: US, Canada, UK, Ireland, Australia and NZ [15, 19, 20, 22-24, 26, 30, 34]. For the 11 apps that could not be found, corresponding authors of the papers were emailed and three replied: one confirming the app is no longer available in app stores [14, 21], one advising the app had been revised and was available under another name [24], and one advising that the app had been temporarily taken down to be updated [17].

Of the 19 unique apps, seven were designed for use among youth [14-21] and 12 in adult populations [22-34]. The results of these studies are summarised in Table 1.

\section{Apps for young people}

The available evidence for the efficacy of alcohol reduction apps among youth is inconclusive, with results from these evaluations not showing a clear benefit in reducing alcohol consumption compared to control groups [1421]. Randomised control trials (RCTs) were conducted for five of the seven apps [14-17, 19, 21], with only two of these finding any significant reductions in alcohol consumption outcomes in intervention compared to control groups $[16,21]$. Of the other two app studies, one was a quasi-experimental, pre-post test design without a control group and found a statistically significant reduction in the proportion of participants reporting binge drinking after the two-week study period compared to baseline $(\mathrm{OR}=0.45,95 \% \mathrm{CI}=0.37-0.55)$ [18]. The other, a cluster non-randomized controlled trial, found no statistically significant difference in past month alcohol use between the intervention and control groups $(\mathrm{P}=0.014)[20]$.

\section{Apps for adult populations}

Of the 12 apps designed for use in adult populations that have been evaluated, four are aimed at individuals in the 


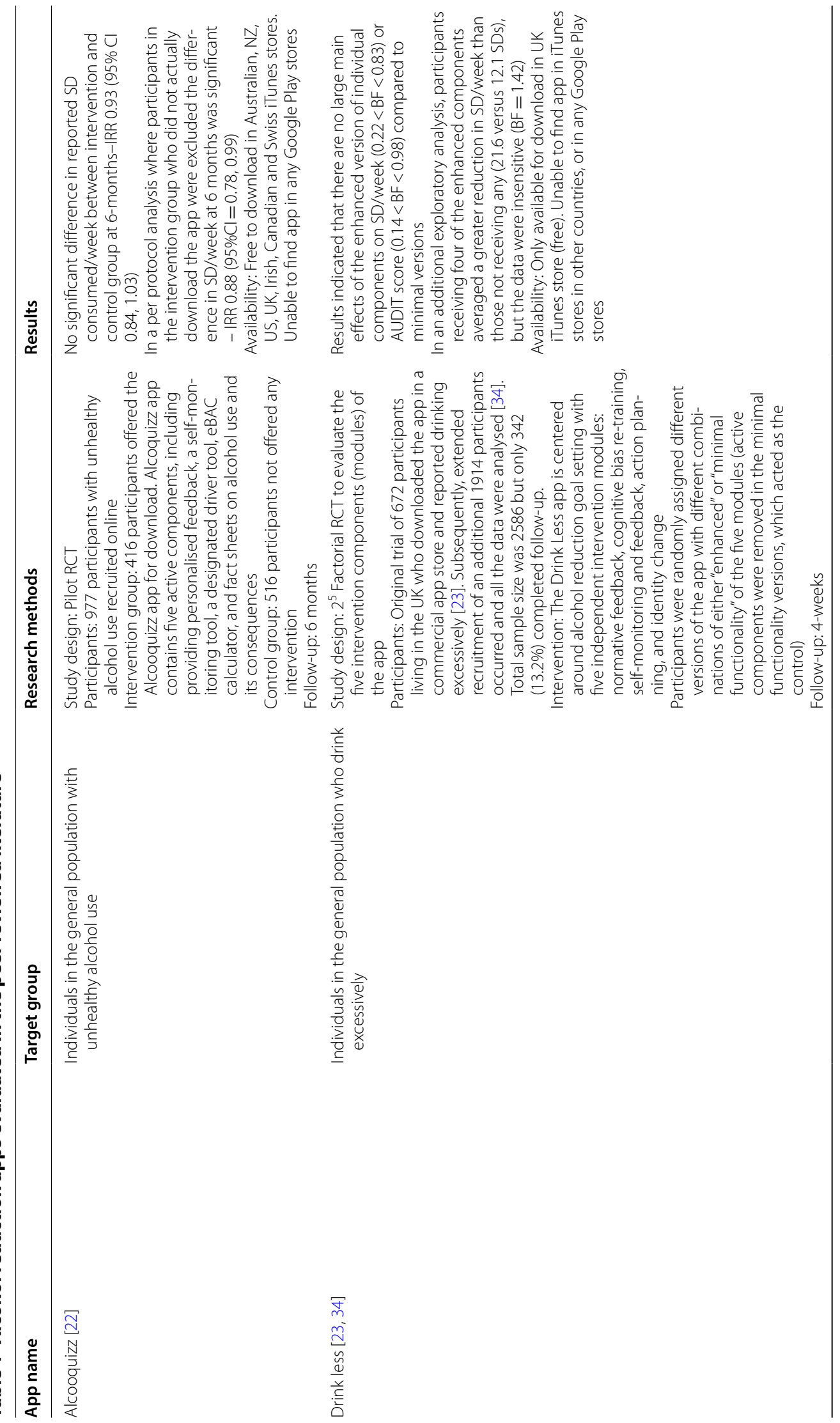




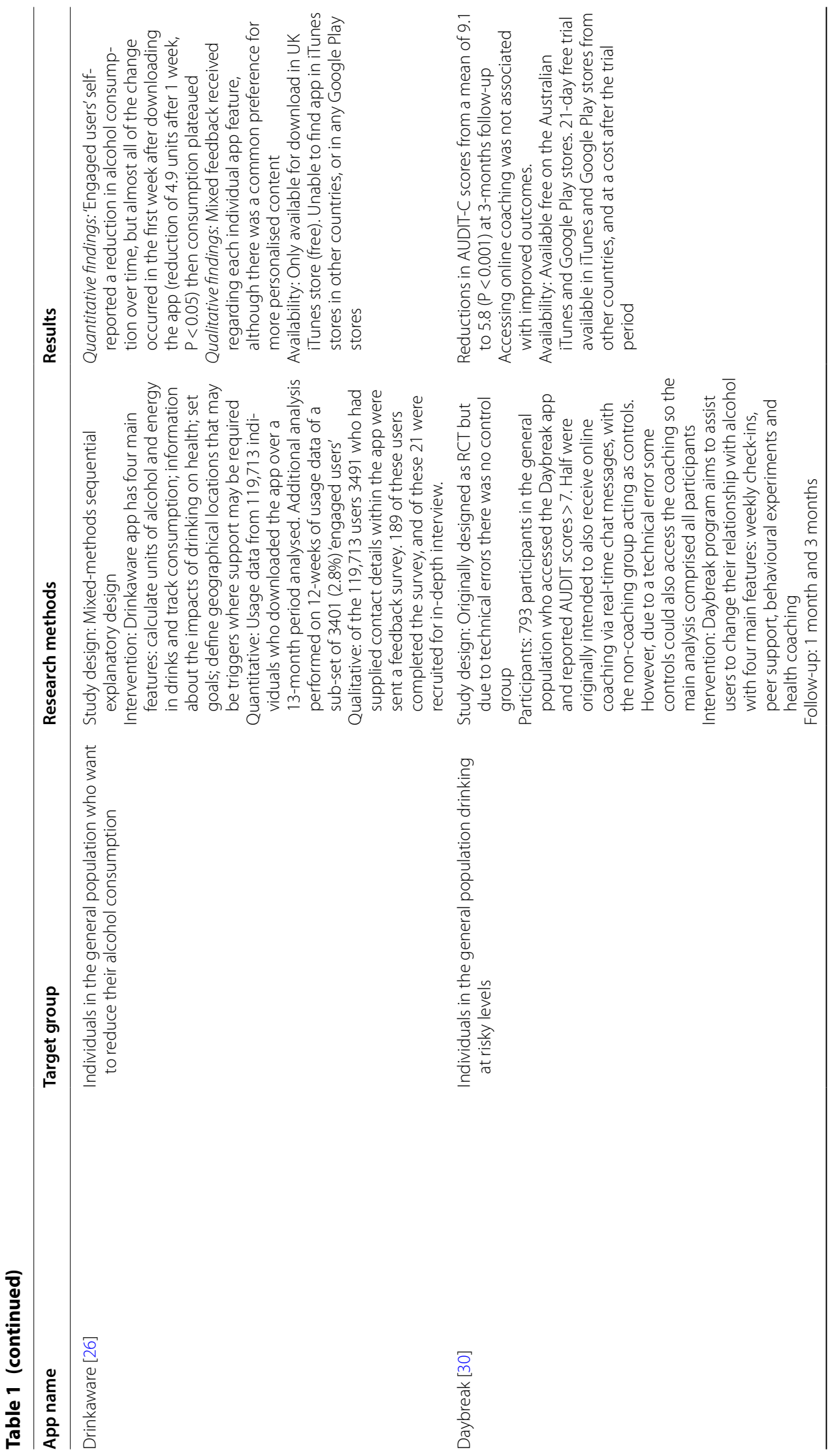




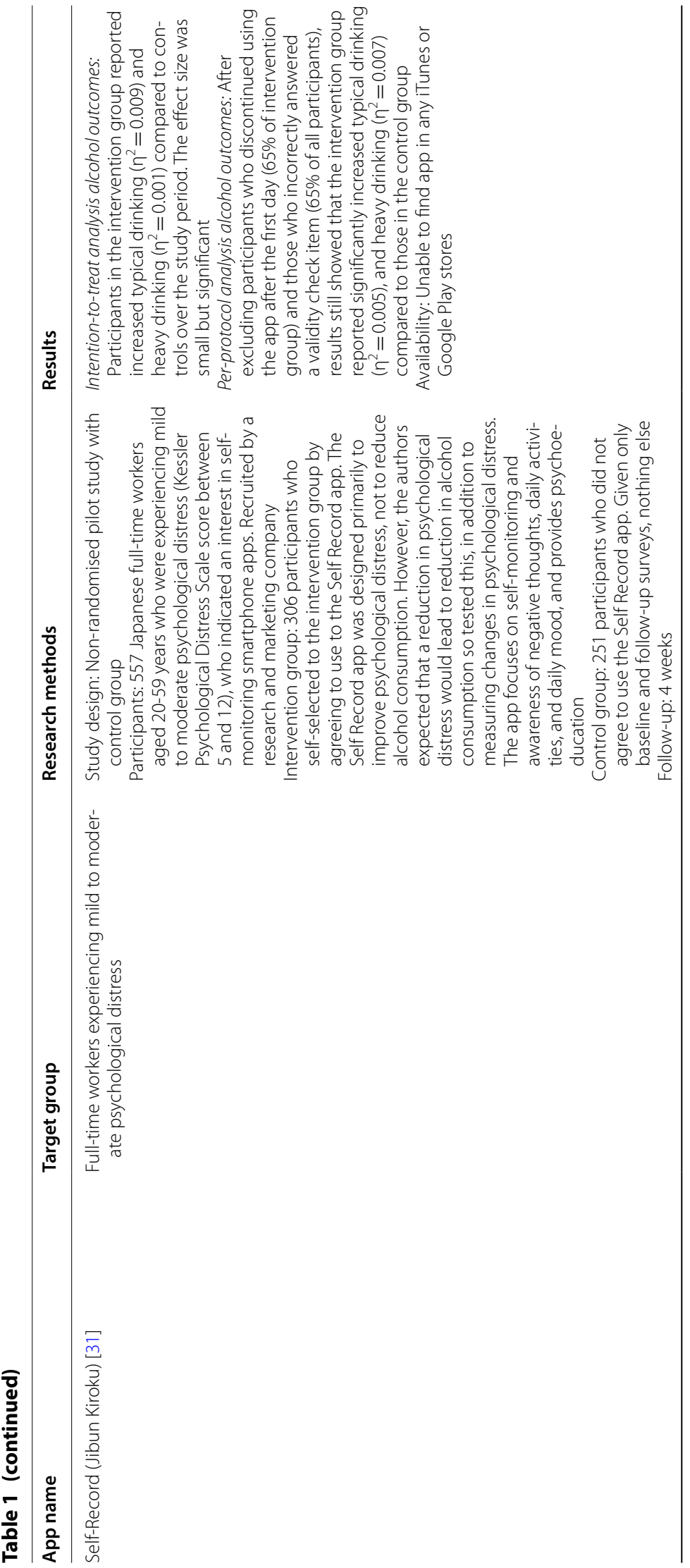




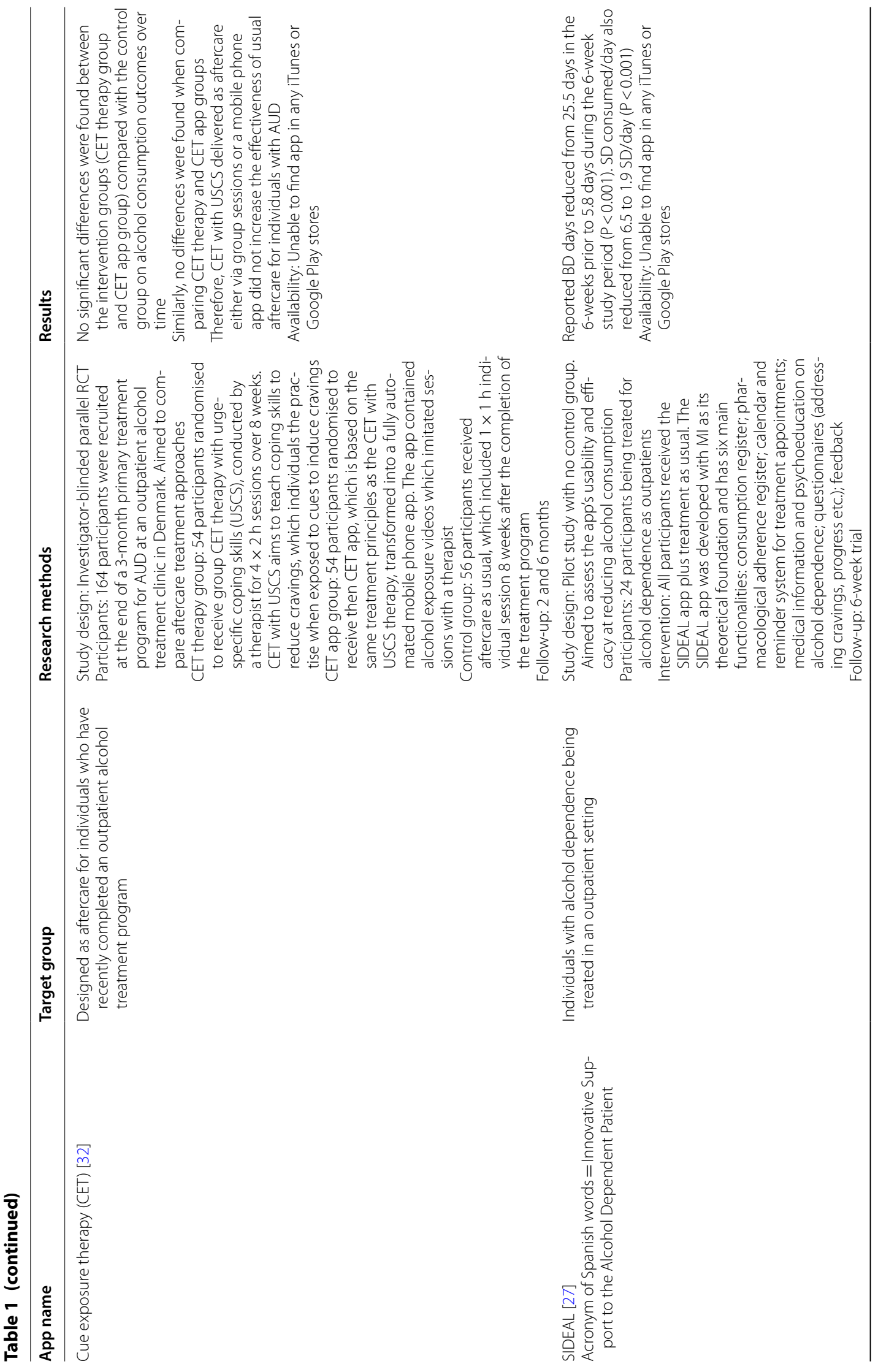




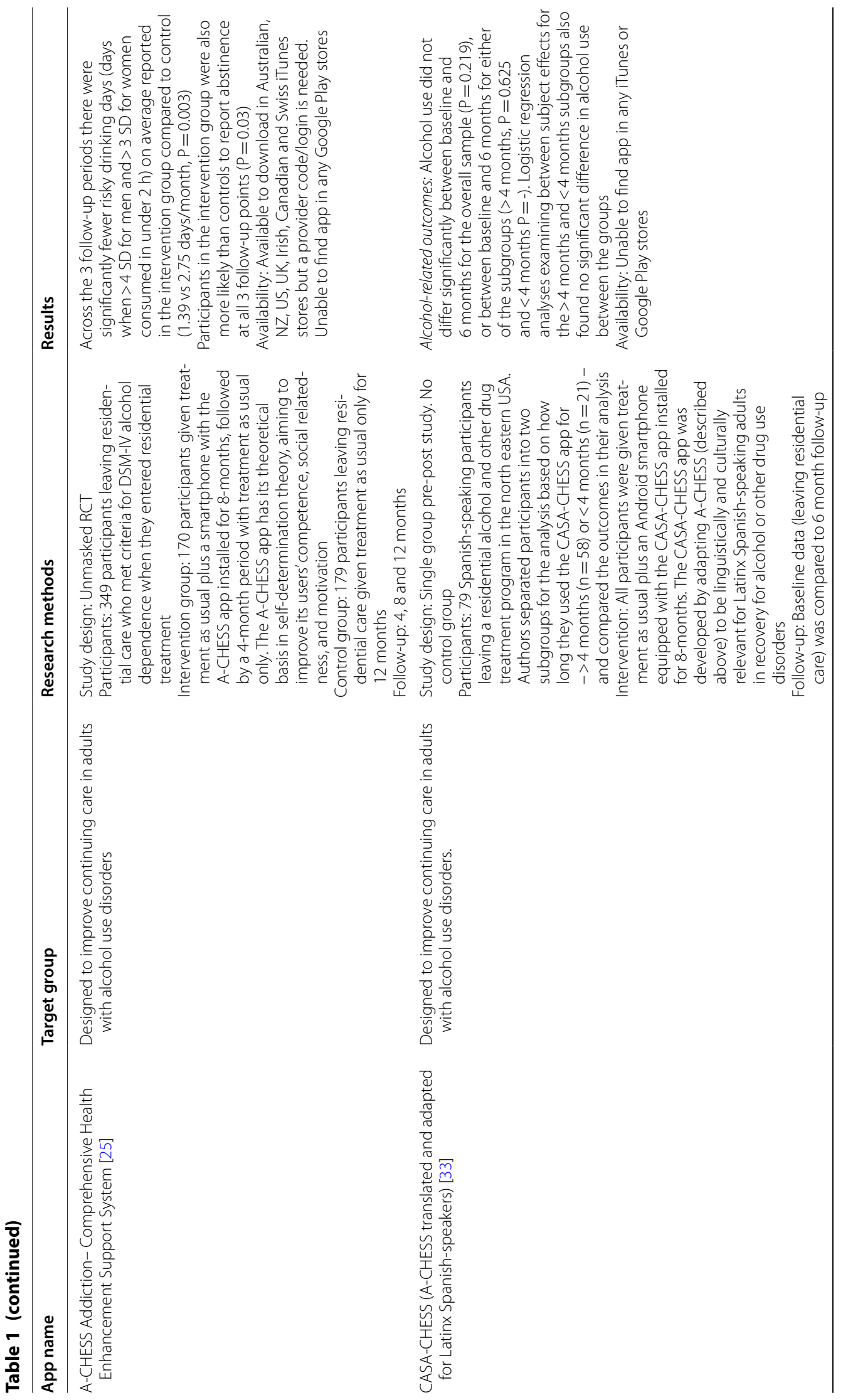




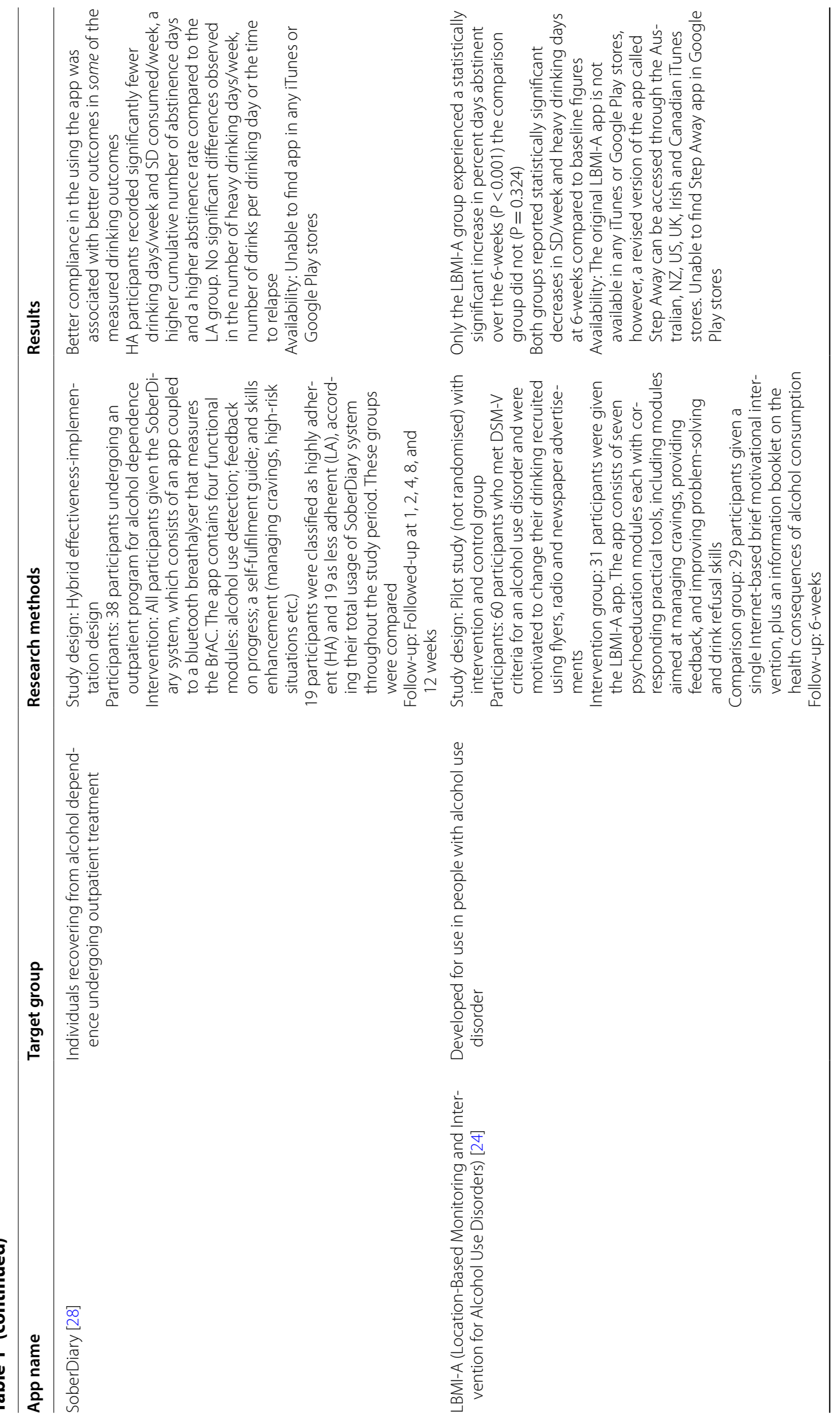




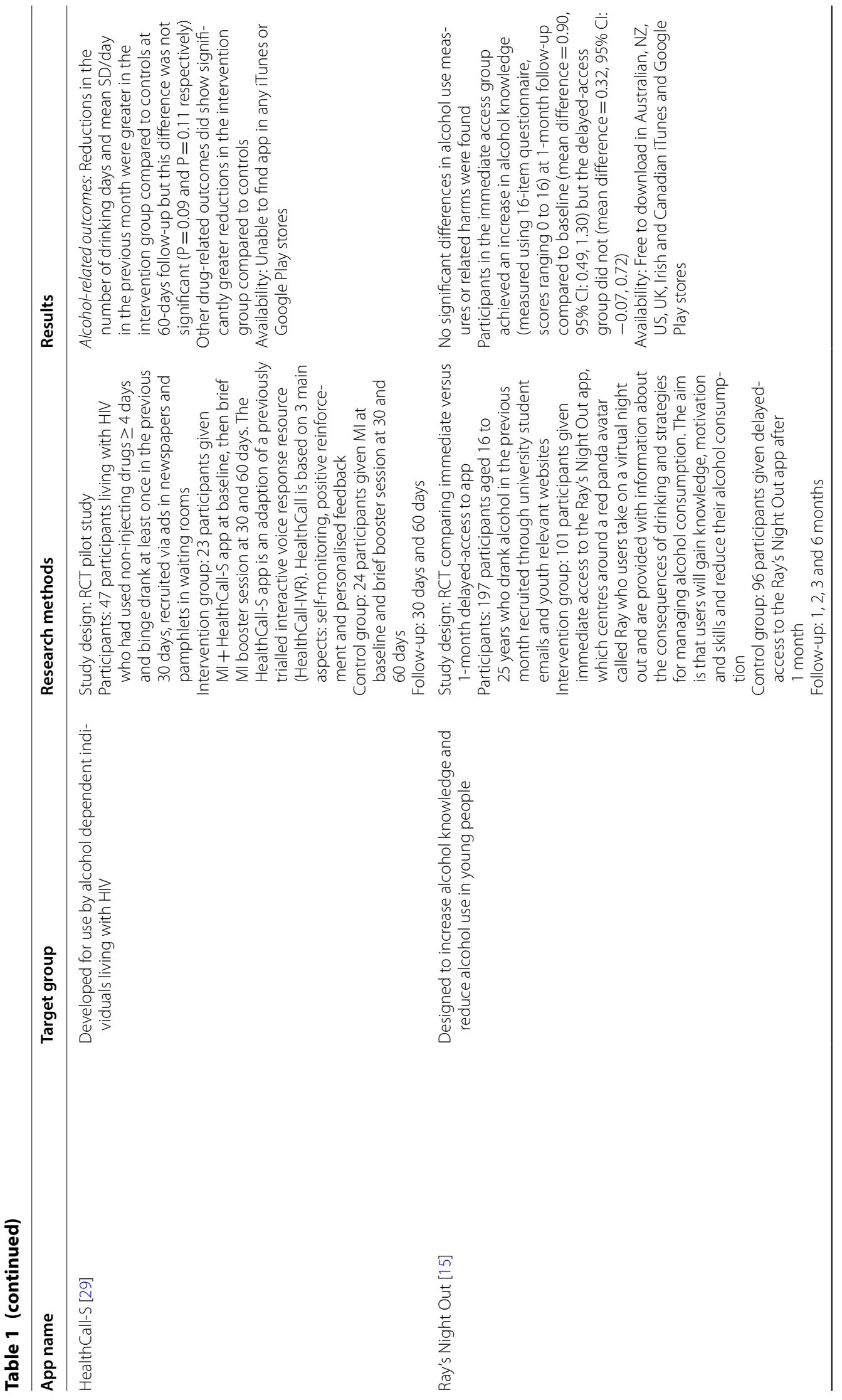




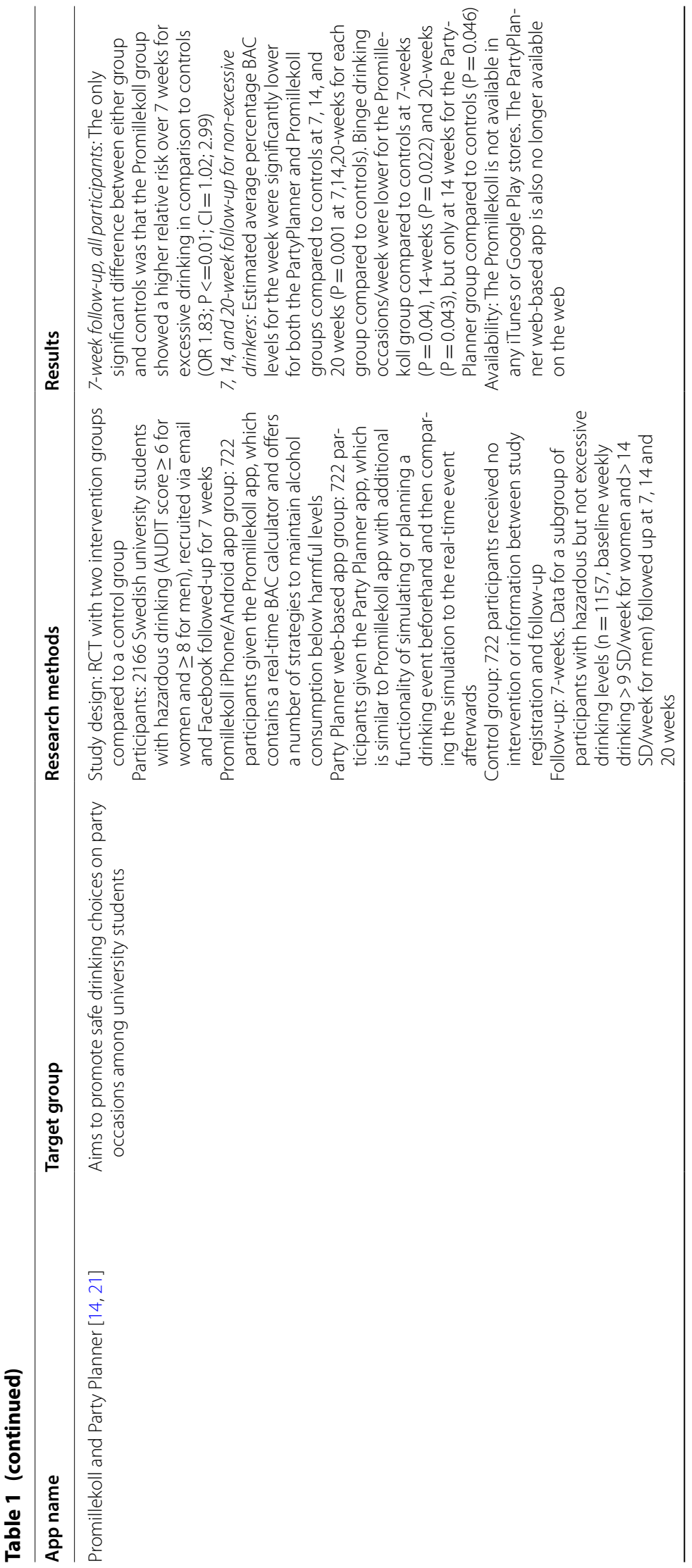




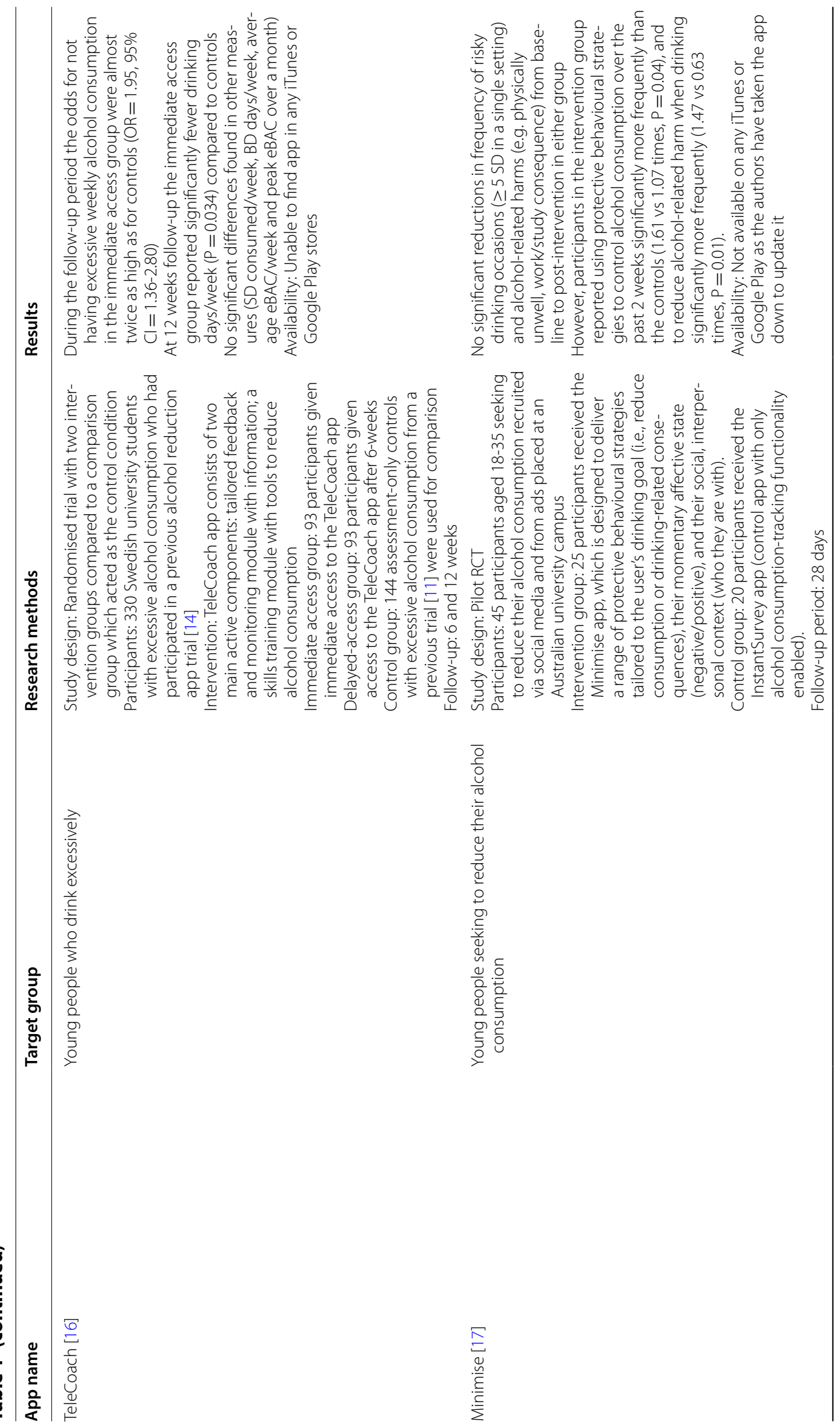




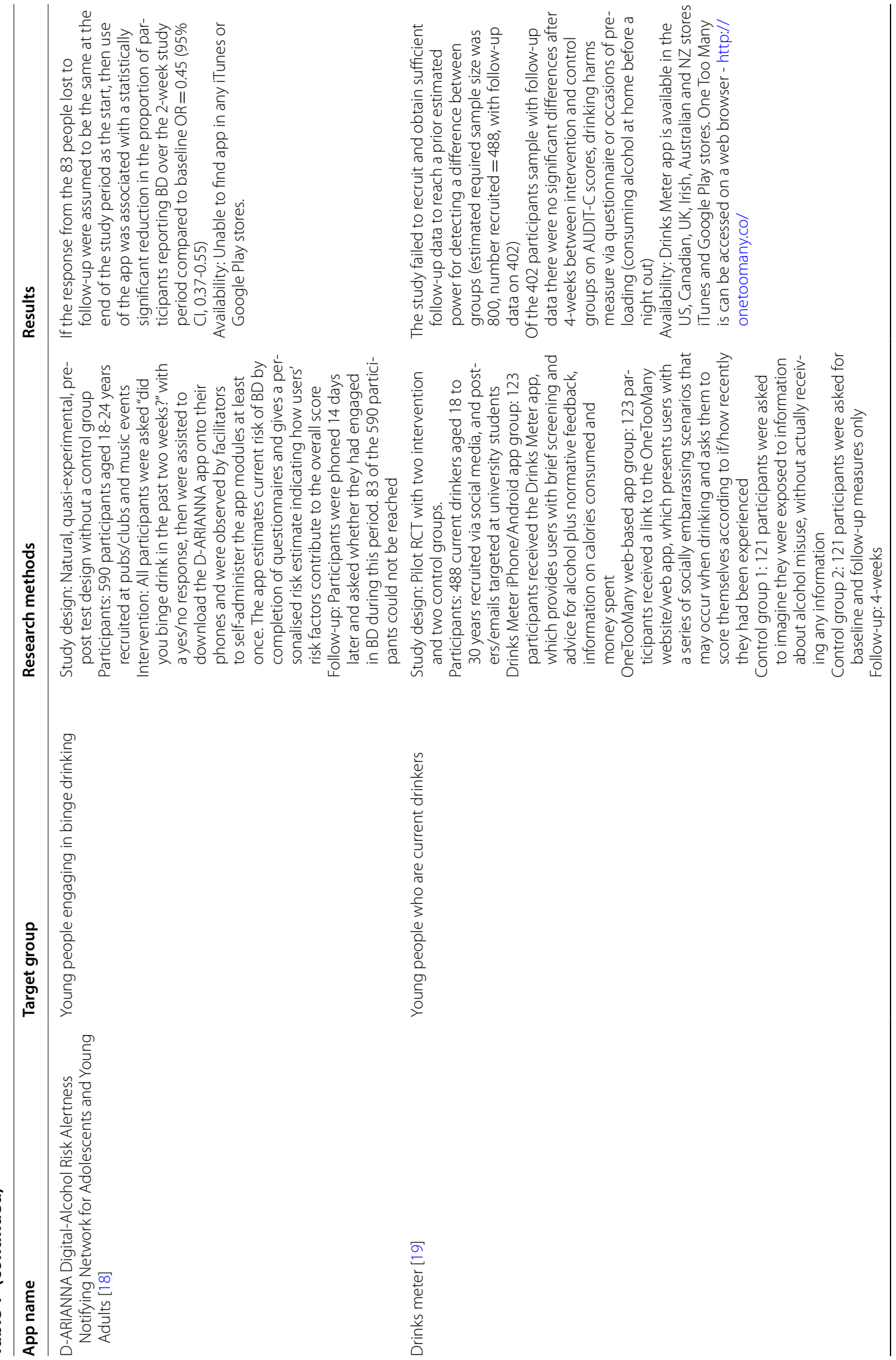




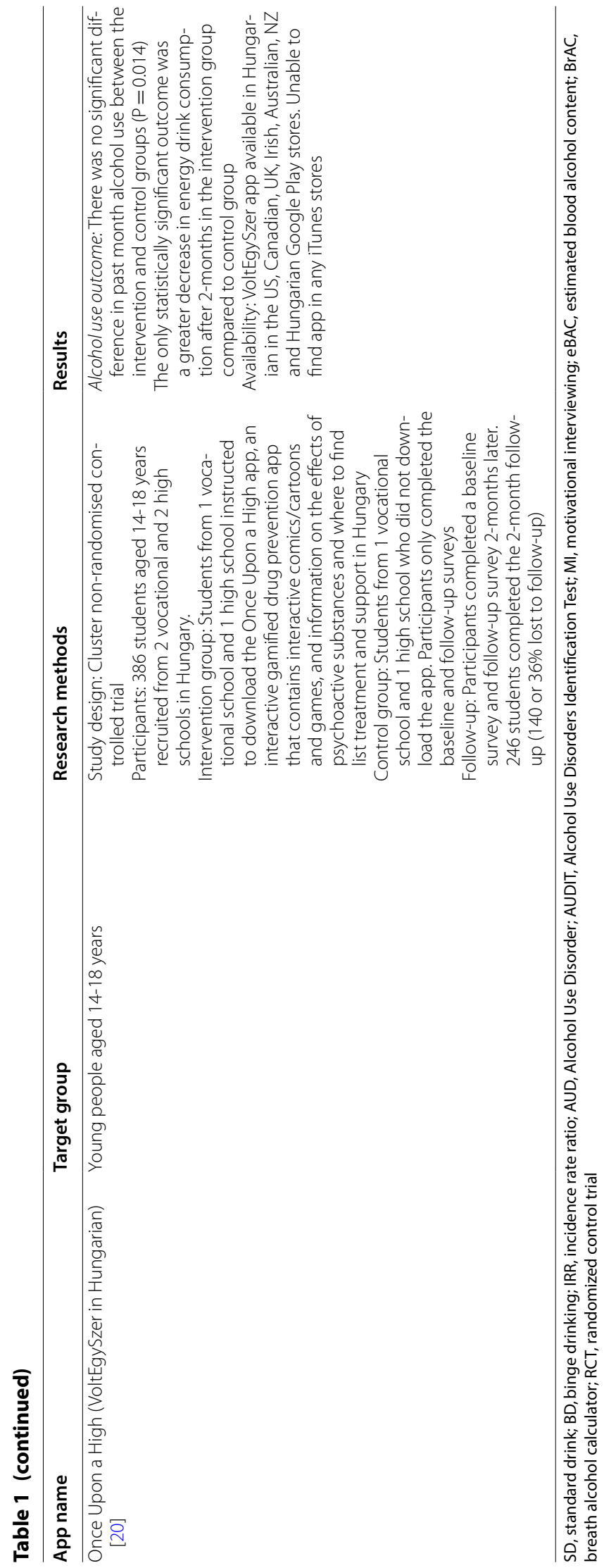


general population who want to reduce their alcohol intake $[22,23,26,30]$, one at working adults experiencing psychological distress [31], and seven at individuals with an alcohol use disorder (AUD) [24, 25, 27-29, 32, 33]. The results from these evaluations are more promising than the apps designed for youth, but results are still mixed. Seven out of 12 studies found significant reductions in alcohol consumption measures, four found no significant reductions and one found a significant increase in alcohol consumption in the intervention group. However, few of these trials were randomized controlled trials with sufficient sample sizes.

Of the four apps aimed at individuals in the general population, two RCTs have been conducted: one found significantly larger reductions in standard drinks consumed per week among participants in one of the app intervention groups compared to controls who were offered the same app with 'active' components removed $(\mathrm{P}=0.03)$ [23]; one did not find a significant difference in standard drinks consumed per week between participants who were offered access to an app and controls who did not receive an intervention $(\mathrm{P}=0.17)$ [22]. Of the other two trials without control groups, one found a significant reduction in Alcohol Use Disorders Identification Test-C (AUDIT-C) scores $(\mathrm{P}<0.001)$ at 3 -months follow-up [30], and the other found a significant reduction in alcohol consumption only among 'engaged app users' [26].

Of the seven apps for use among individuals with AUD there have been three RCTs. One found a significant reduction in 'risky drinking days' among participants using an app compared to controls given only treatment as usual $(\mathrm{P}=0.003)$ [25], while the other two trials found no significant differences in any alcohol consumption outcomes between the intervention and control groups $[29,32]$. The other four trials in this population had mixed results, with three finding significant reductions in alcohol consumption measures and one finding no significant changes.

\section{Availability of apps in commercial app stores}

Of the 19 alcohol reduction apps that have been evaluated [14-34] as far as we can ascertain only eight of these are currently publicly available in commercial app stores $[15,19,20,22-24,26,30,34]$. Of these eight apps, only four were demonstrated in the literature to lead to a reduction in alcohol consumption measures $[23,24,26$, $30,34]$. Two of these apps are exclusively available in the United Kingdom (UK) iTunes stores [23, 34], one is available in the Australian, New Zealand, United States, UK, Irish and Canadian iTunes stores [24], and the other can be accessed from the aforementioned countries' iTunes and Google Play stores but comes at a cost after a 21-day trial period (except for Australian users) [30].

\section{Discussion}

Despite the limited number of apps for managing alcohol consumption that have been evaluated in the literature, and the mixed evidence for their efficacy, there are currently hundreds of alcohol-reduction apps publicly available in the top commercial apps stores iTunes and Google Play. The situation is similar for other health issues, such as increasing physical activity [35]. There are hundreds of apps for increasing physical activity, however few are founded in an evidence-base [35].

\section{Commercial app store content analyses}

Health researchers have begun to conduct content analyses of commercial app stores in order to identify the highest quality publicly available apps in these stores that could potentially be recommended to health professionals and consumers (e.g. [36-38]). These types of analyses involve conducting systematic searches of commercial apps stores to identify publicly available apps purporting to target the issue of interest. Researchers then download the apps and assess them on various quality measures. While this approach is unable to generate evidence regarding the effectiveness of apps, it is a potentially useful method to identify apps that adhere most closely to current evidence-based guidelines and are of the highest technical quality.

There have been several content analyses that have reviewed publicly available apps for managing alcohol consumption [39-44], however most do not identify the apps they found to be the highest quality during their analysis specifically by name meaning a reader could not take advantage of these reviews to locate and use the apps identified as the best. One content analysis that does identify the highest quality apps by name is a 2019 study by Tofighi et al., who reviewed the iTunes and Google Play stores for free or low-cost apps claiming to target alcohol or other substance use and evaluated the apps' functionality, aesthetics, and quality of information of using the validated Mobile App Rating Scale (MARS) [44]. The analysis identified the highest rated apps on the scale and provided descriptions and background information on each app [44]. Content analyses such as this could be useful for health professionals and consumers to narrow down the highest quality apps from the hundreds available, and supplement the lack of evidence in this area.

While evidence for the efficacy of apps to reduce alcohol consumption is still in its infancy, and initial results appear to be mixed, established evidence on digital health interventions more broadly does appear to show a benefit 
in reducing alcohol consumption over those receiving no intervention [10]. However, with the paucity of rigorously evaluated apps clinicians could understandably feel hesitant in recommending them to patients. This issue is common for all health-related smartphone applications, with some healthcare professionals and consumers calling for app regulation or certification to be put in place to ensure quality [45]. In lieu of regulation or greater evidence, several groups have developed checklists that aim to assist in assessing the quality of health apps $[46,47]$. The UK Royal College of Physicians (RCP) Health Informatics Unit has produced a checklist for clinicians to assess the structure, functions, impact and overall quality of health apps [48]. The RCP checklist is designed to help clinicians to feel more confident about recommending health apps to patients [48].

\section{Conclusion}

The evidence for alcohol reduction apps is promising but inconclusive. Few apps that have been evaluated in the scientific literature are currently available for download in commercial app stores. In the absence of more scientifically evaluated apps for reducing alcohol consumption, well-designed content analyses of commercial app stores could potentially assist in identifying the highest quality apps that are publicly available.

\section{Abbreviations}

AUD: Alcohol Use Disorder; AUDIT: Alcohol Use Disorders Identification Test; RCT: Randomised control trials; SD: Standard drink; BD: Binge drinking; IRR: Incidence rate ratio; MI: Motivational interviewing; eBAC: Estimated blood alcohol content; BrAC: Breath alcohol calculator.

\section{Acknowledgements}

Not applicable.

\section{Authors' contributions}

All authors contributed conception of the work. SC reviewed the literature and wrote the first draft. LT and RR reviewed the first draft and substantially revised this and subsequent drafts. All authors read and approved the final manuscript.

\section{Funding}

No funding.

\section{Availability of data and materials \\ Not applicable.}

Ethics approval and consent to participate

Not applicable.

\section{Consent for publication}

Not applicable.

\section{Competing interests}

The authors declare that they have no competing interests.

\section{Author details}

1 School Public Health and Community Medicine, University of New South Wales, Kensington, Australia. ${ }^{2}$ The Matilda Centre for Research in Mental Health and Substance Use, University of Sydney, Sydney, Australia.

Received: 11 September 2019 Accepted: 27 April 2020

Published online: 07 May 2020

\section{References}

1. Global status report on alcohol and health 2018. Geneva: World Health Organization; 2018. https://www.who.int/substance_abuse/publications/ global_alcohol_report/en/.

2. Alcohol, tobacco \& other drugs in Australia (https://www.aihw.gov.au/ reports/alcohol/alcohol-tobacco-other-drugs-australia/contents/drugtypes/alcohol) Accessed 1 June 2019.

3. Substance Abuse and Mental Health Services Administration (SAMHSA). 2018 National Survey on Drug Use and Health (NSDUH). http://www. samhsa.gov/data/release/2018-national-survey-drug-use-and-healt h-nsduh-releases. Accessed 5 March 2020.

4. Kaner EFS, Beyer FR, Muirhead C, Campbell F, Pienaar ED, Bertholet N, Daeppen JB, Saunders JB, Burnand B. Effectiveness of brief alcohol interventions in primary care populations. Cochrane Database of Syst Rev. 2018;2:CD004148.

5. Johnson M, Jackson R, Guillaume L, Meier P, Goyder E. Barriers and facilitators to implementing screening and brief intervention for alcohol misuse: a systematic review of qualitative evidence. J Public Health. 2010:33:412-21.

6. Bearnot B, Rigotti NA, Baggett TP. Access to treatment for alcohol use disorder at US health centers: a national study. J Gen Intern Med. 2018;33:2040-2.

7. Riper H, Spek V, Boon B, Conijn B, Kramer J, Martin-Abello K, Smit F. Effectiveness of E-self-help interventions for curbing adult problem drinking: a meta-analysis. J Med Internet Res. 2011:13:e42

8. Khadjesari Z, Murray E, Hewitt C, Hartley S, Godfrey C. Can stand-alone computer-based interventions reduce alcohol consumption? a systematic review. Addiction. 2011;106:267-82.

9. Carey KB, Scott-Sheldon LAJ, Elliott JC, Bolles JR, Carey MP. Computerdelivered interventions to reduce college student drinking: a meta-analysis. Addiction. 2009;104:1807-19.

10. Kaner E, Beyer F, Garnett C, Crane D, Brown J, Muirhead C, Redmore J, O'Donnell A, Newham J, de Vocht F, et al. Personalised digital interventions for reducing hazardous and harmful alcohol consumption in community-dwelling populations. Cochrane Database Syst Rev. 2017:9:CD011479.

11. The growing value of digital health evidence and impact on human health and the healthcare system (https://www.iqvia.com/institute/repor ts/the-growing-value-of-digital-health Accessed 1 June 2019.).

12. Pew Research Center. Record shares of Americans now own smartphones, have home broadband (http://www.pewresearch.org/facttank/2017/01/12/evolution-of-technology/).

13. Mobile Consumer Survey 2018: The Australian cut (https://www2.deloi tte.com/au/mobile-consumer-survey Accessed 1 June 2019).

14. Gajecki M, Berman AH, Sinadinovic K, Rosendahl I, Andersson C. Mobile phone brief intervention applications for risky alcohol use among university students: a randomized controlled study. Addict Sci Clin Pract. 2014;9:11.

15. Hides L, Quinn C, Cockshaw W, Stoyanov S, Zelenko O, Johnson D, Tjondronegoro D, Quek L-H, Kavanagh DJ. Efficacy and outcomes of a mobile app targeting alcohol use in young people. Addict Behav. 2018;77:89-95.

16. Gajecki M, Andersson C, Rosendahl I, Sinadinovic K, Fredriksson M, Berman $\mathrm{AH}$. Skills training via smartphone app for university students with excessive alcohol consumption: a randomized controlled trial. Int J Behav Med. 2017;24:778-88.

17. O'Donnell R, Richardson B, Fuller-Tyszkiewicz M, Staiger PK. Delivering personalized protective behavioral drinking strategies via a smartphone intervention: a pilot study. Int J Behav Med. 2019;26:401-14.

18. Carrà G, Crocamo C, Bartoli F, Carretta D, Schivalocchi A, Bebbington PE, Clerici M. Impact of a mobile e-health intervention on binge drinking 
in young people: the digital-alcohol risk alertness notifying network for adolescents and young adults project. J Adolesc Health. 2016;58:520-6.

19. Davies EL, Lonsdale AJ, Hennelly SE, Winstock AR, Foxcroft DR. Personalized digital interventions showed no impact on risky drinking in young adults: a pilot randomized controlled trial. Alcohol Alcohol. 2017;52:671-6.

20. Kapitány-Fövény M, Vagdalt E, Ruttkay Z, Urbán R, Richman MJ, Demetrovics Z. Potential of an interactive drug prevention mobile phone app (once upon a high): questionnaire study among students. JMIR Serious Games. 2018;6:e19.

21. Berman AH, Andersson C, Gajecki M, Rosendahl I, Sinadinovic K, Blankers M. Smartphone apps targeting hazardous drinking patterns among university students show differential subgroup effects over 20 weeks: results from a randomized, controlled trial. J Clin Med. 1807;2019:8.

22. Bertholet N, Godinho A, Cunningham JA. Smartphone application for unhealthy alcohol use: pilot randomized controlled trial in the general population. Drug Alcohol Depend. 2019;195:101-5.

23. Crane D, Garnett C, Michie S, West R, Brown J. A smartphone app to reduce excessive alcohol consumption: identifying the effectiveness of intervention components in a factorial randomised control trial. Sci Rep. 2018;8:1.

24. Gonzalez VM, Dulin PL. Comparison of a smartphone app for alcohol use disorders with an internet-based intervention plus bibliotherapy: a pilot study. 2015;83:335.

25. Gustafson DH, McTavish FM, Chih M-Y, Atwood AK, Johnson RA, Boyle MG, Levy MS, Driscoll H, Chisholm SM, Dillenburg L, et al. A smartphone application to support recovery from alcoholism: a randomized clinical trial. JAMA Psychiatry. 2014;71:566-72.

26. Attwood S, Parke H, Larsen J, Morton KL. Using a mobile health application to reduce alcohol consumption: a mixed-methods evaluation of the drinkaware track \& calculate units application. BMC Public Health. 2017:17:394.

27. Barrio P, Ortega L, López H, Gual A. Self-management and Shared decision-making in alcohol dependence via a mobile app: a pilot study. Off J Int Soc Behav Med. 2017;24:722-7.

28. You C-W, Chen Y-C, Chen C-H, Lee C-H, Kuo P-H, Huang M-C, Chu H-H. Smartphone-based support system (SoberDiary) coupled with a Bluetooth breathalyser for treatment-seeking alcohol-dependent patients. Addict Behav. 2017;65:174-8.

29. Aharonovich E, Stohl M, Cannizzaro D, Hasin D. HealthCall delivered via smartphone to reduce co-occurring drug and alcohol use in HIV-infected adults: a randomized pilot trial. J Subst Abuse Treat. 2017;83:15-26.

30. Tait RJ, Paz Castro R, Kirkman JJL, Moore JC, Schaub MP. A digital intervention addressing alcohol use problems (the "daybreak" program): quasi-experimental randomized controlled trial. J Med Internet Res. 2019;21:e14967.

31. Hamamura T, Suganuma S, Ueda M, Mearns J, Shimoyama H. Standalone effects of a cognitive behavioral intervention using a mobile phone app on psychological distress and alcohol consumption among japanese workers: pilot nonrandomized controlled trial. JMIR Mental Health. 2018;5:e24.

32. Mellentin Al, Nielsen B, Nielsen A, Yu F, Mejldal A, Nielsen DG, Stenager E. A mobile phone app featuring cue exposure therapy as aftercare for alcohol use disorders: an investigator-blinded randomized controlled trial. JMIR mHealth uHealth. 2019:7:13793.

33. Muroff J, Robinson W, Chassler D, López LM, Lundgren L, Guauque C, Dargon-Hart S, Stewart E, Dejesus D, Johnson K, et al. An outcome study of the CASA-CHESS smartphone relapse prevention tool for Latinx Spanish-speakers with substance use disorders. Subst Use Misuse. 2019:54:1438-49.

34. Garnett C, Michie S, West R, Brown J. Updating the evidence on the effectiveness of the alcohol reduction app, drink less: using Bayes factors to analyse trial datasets supplemented with extended recruitment. F1000 Res. 2019;8:114

35. Kebede M, Steenbock B, Helmer SM, Sill J, Möllers T, Pischke CR. Identifying evidence-informed physical activity apps: content analysis. JMIR mHealth and uHealth. 2018;6:e10314-e10314.

36. Thornton L, Quinn C, Birrell L, Guillaumier A, Shaw B, Forbes E, Deady $M$, Kay-Lambkin F. Free smoking cessation mobile apps available in Australia: a quality review and content analysis. Aust N Z J Public Health. 2017:41:625-30.

37. Middelweerd A, Mollee JS, van der Wal CN, Brug J, te Velde SJ. Apps to promote physical activity among adults: a review and content analysis. Int J Behav Nutr Phys Act. 2014;11:97.

38. Shen $N$, Levitan M-J, Johnson A, Bender JL, Hamilton-Page M, Jadad AAR, Wiljer $\mathrm{D}$. Finding a depression app: a review and content analysis of the depression app marketplace. JMIR mHealth and uHealth. 2015;3:e16-e16.

39. Crane D, Garnett C, Brown J, West R, Michie S. Behavior change techniques in popular alcohol reduction apps: content analysis. J Med Internet Res. 2015;17:e118-e118.

40. Cohn AM, Hunter-Reel D, Hagman BT, Mitchell J. Promoting behavior change from alcohol use through mobile technology: the future of ecological momentary assessment. Alcohol Clin Exp Res. 2011;35:2209-15.

41. Penzenstadler $L$, Chatton $A$, Van Singer M, Khazaal Y. Quality of smartphone apps related to alcohol use disorder. Eur Addict Res. 2016;22:329-38.

42. Hoeppner BB, Schick MR, Kelly LM, Hoeppner SS, Bergman B, Kelly JF. There is an app for that-or is there? a content analysis of publicly available smartphone apps for managing alcohol use. J Subst Abuse Treat. 2017;82:67-73

43. Weaver ER, Horyniak DR, Jenkinson R, Dietze P, Lim MS. "Let's get wasted!" and other apps: characteristics, acceptability, and use of alcohol-related smartphone applications. JMIR Mhealth Uhealth. 2013;1:e9.

44. Tofighi B, Chemi C, Ruiz-Valcarcel J, Hein P, Hu L. Smartphone apps targeting alcohol and illicit substance use: systematic search in in commercial app stores and critical content analysis. JMIR Mhealth Uhealth. 2019;7:e11831.

45. Boulos MNK, Brewer AC, Karimkhani C, Buller DB, Dellavalle RP. Mobile medical and health apps: state of the art, concerns, regulatory control and certification. Online J Public Health Inform. 2014;5:229-229.

46. Stoyanov SR, Hides L, Kavanagh DJ, Zelenko O, Tjondronegoro D, Mani M. Mobile app rating scale: a new tool for assessing the quality of health mobile apps. JMIR mHealth and uHealth. 2015;3:e27-e27.

47. Wicks P, Chiauzzi E. 'Trust but verify'-five approaches to ensure safe medical apps. BMC Med. 2015;13:205-205.

48. Wyatt JC, Thimbleby H, Rastall P, Hoogewerf J, Wooldridge D, Williams J. What makes a good clinical app? introducing the RCP health informatics unit checklist. Clin Med (London, England). 2015;15:519-21.

\section{Publisher's Note}

Springer Nature remains neutral with regard to jurisdictional claims in published maps and institutional affiliations. 